Alexandra DESTAIS, «L'érographie littéraire féminine : la frénésie sexuelle maîtrisée? », dans J. Delorme et Cl. Labrosse (dir.), «Écriture du corps, corps de l'écriture : la dialectique du corps-écriture dans le roman francophone au XX siècle », @nalyses, hiver 2008

\title{
Alexandra DeSTAIS
}

\section{L'érographie littéraire féminine: la frénésie sexuelle maîtrisée?}

Dans le récit «érographique » (Brulotte, p. 6), le corps est une entité organique qui fait l'objet de deux transformations essentielles. D'une part, le potentiel initial du corps sexuel est exploité jusqu'à son point de rupture, d'où une extension du domaine du vivant; d'autre part, le corps érographique fait de l'expérience charnelle une dynamique autonome qui bouleverse la coïncidence entre le corps et la psyché, d'où une privation de la personne. La circulation d'une force motrice inépuisable au sein de séances érographiques fondées sur le principe de répétition suppose l'élimination d'un certain nombre de contraintes liées à l'économie narrative traditionnelle. Seule une pensée négatrice de la personne permet d'isoler la luxure à l'état pur, de la délivrer du réseau pesant des obstacles sentimentaux, psychologiques et sociaux qui empêchent le libre déploiement instinctuel. Un effet érotique se met ainsi en place, dont l'origine réside non seulement dans le spectacle du corps libertin triomphant, mais aussi dans celui du corps victimal dont les manifestations subjectives sont limitées au cri de la souffrance.

S’il existe un écrivain qui a apporté ses lettres de noblesse à la représentation érographique du corps sexuel et à partir duquel Roland Barthes (1980a), puis Gaëtan Brulotte ont identifié une "passion structurale »(1998a, p. 307), c'est, bien entendu, Sade. Son œuvre a longtemps suscité la réprobation la plus vive en raison notamment de sa remise en cause radicale d'une vision humaniste du corps humain. Le double mouvement d'amplification de la puissance naturelle du corps vivant et de négation de la personne a été porté à son plus haut degré dans Les Cent Vingt Journées de Sodome (Pia, 1998), qui a influencé des générations entières d'érographes. En raison des forces anarchiques et animales qui semblent gouverner les personnages, on pourrait s'attendre à ce que le récit érographique rejette le carcan des règles qui régissent la narration classique au profit d'un libertinage formel. Au 
Alexandra DESTAIS, «L'érographie littéraire féminine : la frénésie sexuelle maîtrisée? », dans J. Delorme et Cl. LABRosse (dir.), «Écriture du corps, corps de l'écriture : la dialectique du corps-écriture dans le roman francophone au XX siècle », @nalyses, hiver 2008

contraire, l'examen de Barthes prouve l'existence d'un principe d'ordre qui organise la matière charnelle. On peut toutefois se demander si ces contraintes s'appliquent au récit érographique féminin.

L'érotisme littéraire féminin a longtemps péché par défaut d'inventivité, selon les principaux commentateurs de cette fraction maudite de la littérature. Selon Alexandrian, les femmes seraient moins aptes que les hommes à transformer en «idées » et en "images » les «sensations» sexuelles (1989, p. 247). Même si, d'après Paulhan, «tout est sexe en elles, et ce, jusqu'à l'esprit»(1975, p. 10), aucun projet véritablement créateur, fondé sur la mise à distance tragique, ludique ou ironique des fantasmes sexuels ou sur la transposition élaborée d'un vécu sulfureux ne semble émerger de la production narrative féminine jusqu'à Histoire d'O (1975). Si l'intérêt pour la vie sexuelle des femmes s'accentue à l'issue de la Seconde Guerre mondiale, comme le montre par exemple le document de vie de Marie-Thérèse (1947) que Simone de Beauvoir soutient dans le cadre des Temps modernes, le traitement de la sexualité s'effectue principalement sur un mode réaliste. Subordonné à un questionnement identitaire et non à la mise en scène érotique, l'élément sexuel est surtout intégré et développé au sein du projet plus vaste du roman revendicatif féminin. De plus, une longue tradition de décence pèse sur les auteures. En vertu d'une émotivité jugée inconciliable avec un travail d'élaboration secondaire, les écrivaines ont été volontiers invitées à entretenir les diverses ramifications intimes du "genre familier ", à l'exception du domaine érographique. Les lettres, les journaux intimes et une attitude autobiographique forment au XIX ${ }^{\mathrm{e}}$ siècle leur domaine de prédilection, le genre érotique étant alors considéré comme " peu conforme à l'idéal moral féminin de modestie et d'humilité » (Plante, p. 227), contrairement au roman d'amour dont elles semblent les plus ardentes ambassadrices. Jusqu'à la parution d'Histoire d'O en 1954, peu d'auteures osent enfreindre cette règle d'exclusion implicite propre à la «catégorisation sexuelle de la littérature» (Plante, p. 231). Moins capables de sublimation, elles seraient aussi moins rigoureuses au plan formel, préférant à la préparation laborieuse la spontanéité de l'épanchement scriptural. 
Alexandra DESTAIS, «L'érographie littéraire féminine : la frénésie sexuelle maîtrisée? », dans J. Delorme et Cl. Labrosse (dir.), «Écriture du corps, corps de l'écriture : la dialectique du corps-écriture dans le roman francophone au XX siècle », @nalyses, hiver 2008

Dans le récit sadien, il existe une complicité d'ordre homologique entre l'organisation de l'intrigue, le mode d'exposition du récit, les procédés d'écriture et une certaine représentation du corps érographique. Au corps fragmenté, coupé de ses ressorts psychologiques, mécanisé, correspondent une organisation rationnelle du texte, une narration objective et une parole libertine stéréotypée (Castillo Durante). Cette performativité est-elle reconduite chez les érographes féminins? Une certaine économie formelle soutient-elle une approche différente du corps érographique?

\section{Le corps érotico-amoureux d'O : un récit érographique?}

La représentation érographique du corps sadien diffère très largement de l'image lyrique du corps amoureux. Elle est même son antithèse, le libertin dénonçant volontiers l'abus de sentimentalité qui compromet l'accès à la jouissance la plus vive. Dans un schéma romanesque classique, comme celui d'Anna Karénine, le corps est un foyer vital de tension amoureuse vers lequel converge tout ce que la parole pétrifiée propre à une société conventionnelle soucieuse de bienséance est incapable de prendre en charge. Il est le lieu qui cache et dévoile dans un même mouvement l'appel téméraire de l'amour passionnel et les clivages internes qui retardent son aveu ainsi que sa pleine expansion. Il accueille, concentre et problématise ce que le corps libertin décomplexé extériorise bruyamment. Le corps libertin met toutes ses facultés motrices et énergétiques au service d'une "décharge », c'est-àdire d'une perte orgasmique sans supplément d'âme. À cette dimension fantastique d'un corps-machine qui ne se soumet pas aux limites physiologiques, qui entretient sa «force»par des mesures extraordinaires, qui fait parade de son appétit orgiaque, Histoire d'O oppose un corps dont l'héroïsme réside dans sa faculté de résistance aux sévices sadomasochistes qui l'aliènent, l'humilient, le réduisent.

Le corps d'O est soumis à des contraintes externes qui visent à tuer sa part non maitrisable, à le renvoyer "par la force aux jours où le désir ne s'était pas déclaré encore » (1975, p. 13). Au terme d'une initiation qui s'étend sur quatre saisons, l'hérö̈ne devient un animal domestiqué, comme le montre son déguisement de chouette chevêche à la fin du 
Alexandra DESTAIS, «L'érographie littéraire féminine : la frénésie sexuelle maîtrisée? », dans J. DelORME et Cl. LABROSSE (dir.), «Écriture du corps, corps de l'écriture : la dialectique du corps-écriture dans le roman francophone au XX siècle », @nalyses, hiver 2008

récit (p. 199). Tandis que le corps d'O est un corps thématique - et non anecdotique (Berthelot, p. 27) — autour duquel s'articule l'histoire, il est soumis à un processus d'effacement. Son corps finit par n'exister qu'à travers l'usage qu'en font les hommes auprès desquels il circule ou face auxquels il est exposé comme un « objet de démonstration » (p. 200). Comme c'est le cas dans la plupart des récits érographiques, la réalité physique d'O se manifeste moins comme un corps total que comme une réalité réduite à une érotique d'organes. Réduit à un composite de lieux-clefs (le sexe, la bouche, l'anus) où s'exerce le pouvoir phallique, le corps d'O est un corps volontairement mis sous tutelle, que la communauté des maitres de Roissy s'approprie au détriment de la personne dont l'autonomie physique est supprimée :

\footnotetext{
Vos mains ne sont pas à vous, ni vos seins, ni tout particulièrement aucun des orifices de votre corps, que nous pouvons fouiller et dans lesquels nous pouvons nous enfoncer à notre gré. Par manière de signe, pour qu'il vous soit constamment présent à l'esprit, ou aussi présent que possible, que vous avez perdu le droit de vous dérober, devant nous vous ne fermerez jamais tout à fait les lèvres, ni ne croiserez les jambes, ni ne serrerez les genoux [...] votre bouche, votre ventre, et vos reins nous sont ouverts. (p. 37)
}

Dénaturé comme le montre la double transformation, superficielle (recours aux fards, au parfum, aux vêtements) et profonde (élargissement de l'anus, marque au fer rouge, etc.), qu'il subit, le corps d'O demeure néanmoins un corps vivant qui souffre ou qui jouit. Il est le support de sensations et d'émotions qui n'engagent pas uniquement l'une de ses parties, mais sa totalité sensorielle: "elle sentait la chaleur, et entendait crépiter doucement les bûches dans le silence» (p. 31). Car la représentation du corps d'O n'hérite pas seulement du postulat matérialiste où est privilégié le triptyque bouche-sexe-anus. Objet consentant, l'héroïne est aussi une "fille amoureuse » (Réage, 1969, p. 205) dotée d'un corps qui signifie. Expressif, il est le lieu où s'affiche le conflit intérieur entre les données physiques et psychologiques qui entravent la quête «mystique» de l'héroïne (orgueil, souffrance) et la «volonté » "fanatique » (Réage, 1975, p. 21) de continuer malgré tout afin que «ne s'efface pas la grâce » (p. 110) d'être aimée. Ainsi, la réduction à la substance intervient au prix d'un arrachement de la personne sociale, mais non 
Alexandra DESTAIS, «L'érographie littéraire féminine : la frénésie sexuelle maîtrisée? », dans J. DelORME et Cl. LABROSSE (dir.), «Écriture du corps, corps de l'écriture : la dialectique du corps-écriture dans le roman francophone au XX siècle », @nalyses, hiver 2008

pas de l'intériorité psychologique. L'héroïne suscite un effet de réel, non seulement à travers son corps sensible engagé dans une dynamique sexuelle, mais aussi à travers l'activité de sa conscience lors de pauses narratives où elle interroge les données de l'action. Car contrairement à un récit érographique tel Les Onze Mille verges, il ne s'agit pas de mentionner brièvement un retour de la conscience sur elle-même (1973, p. 95) afin d'amorcer une nouvelle séquence érographique, mais d'énoncer les termes d'une pensée en mouvement, avide de « comprendre » :

Couchée sur le côté gauche, et seule dans le noir et le silence, chaude entre ses deux épaisseurs de fourrure, et par force immobile, $\mathrm{O}$ se demandait pourquoi tant de douceur se mêlait en elle à la terreur, ou pourquoi la terreur lui était si douce [...]. Elle ne sentait plus que le collier, les bracelets et la chaîne, son corps partait à la dérive, elle allait comprendre. Elle s'endormit. (p. 45)

Le statut mixte d'un corps érotico-amoureux, à la fois prétexte à la mise en place de la séance érographique et enjeu d'une histoire qui le détruit peu à peu, ne conditionne pas seulement une succession de scènes sexuelles à peine reliées par un fil narratif ténu, mais également un crescendo narratif qui permet de rendre compte des "progrès d'une femme » (Mandiargues) en même temps que de son glissement progressif vers un dénouement tragique. À la fois récit érographique et «farouche lettre d'amour» (Réage, 1975, p. 17), Histoire d'O illustre la conciliation entre deux types de contraintes : celles propres au genre romanesque, où la mise en œuvre d'une intrigue forme une histoire, et celles propres au genre érographique.

\section{La complicité homologique entre représentation du corps et contraintes formelles}

Sur le plan scénique, l'accès à la conscience d'O est favorisé par le positionnement de son corps dans l'espace. À plusieurs reprises, l'immobilité corporelle entraîne le relais du point de vue externe par une focalisation interne qui ouvre l'accès au sujet psychologique. Par exemple, au début du récit, la contrainte que subit $O$ en étant enchaînée au mur de son lit par un valet de Roissy engendre un 
Alexandra DESTAIS, «L'érographie littéraire féminine : la frénésie sexuelle maîtrisée? », dans J. DelORME et Cl. LABROSSE (dir.), «Écriture du corps, corps de l'écriture : la dialectique du corps-écriture dans le roman francophone au XX siècle », @nalyses, hiver 2008

monologue narrativisé à tonalité non ironique. Nous découvrons ainsi les sentiments contradictoires de l'héroïne :

On l'avait délivrée de ses mains; son corps sous la fourrure lui était à ellemême inaccessible; que c'était étrange de ne pouvoir toucher ses propres genoux, ni le creux de son propre ventre [...]. Elle glissa un peu sur le ventre, songea que son amant aimait le sillon de ses reins, qu'à la réserve de ce soir (si c'était lui) il n'avait jamais pénétré. Elle souhaita que c'eût été lui; lui demanderait-elle? Ah! Jamais. (p. 44)

De même, dans une scène de «Sir Stephen », tandis qu'O doit demeurer immobile dans une posture d'ouverture sexuelle, la voix narrative nous permet non seulement de recevoir des bribes indirectes de la conversation entre René et Sir Stephen, mais surtout d'investir les pensées d'O :

[Elle] se retrouva couchée sur le dos par le travers de la table, au milieu des photos, où Sir Stephen, la quittant, l'avait brusquement jetée, les jambes écartées et pendantes. Ses pieds ne touchaient pas terre, une de ses mules lui échappa, glissa sans bruit sur le tapis blanc. [...] elle assista alors au dialogue entre Sir Stephen et René, ainsi gisante, comme s'il ne la concernait pas [...]. O écoutait, toujours renversée et brûlante et immobile, et il lui semblait que Sir Stephen, par une étrange substitution, parlait pour elle, et à sa place. (p. 127)

Le caractère anecdotique d'un corps inemployé offre soudain à $\mathrm{O}$ l'occasion d'endosser le rôle de témoin discret d'une conversation dont elle est l'objet au sein d'une pause narrative orientée vers l'évocation empathique des sentiments du personnage.

Histoire d'O reconduit certaines contraintes de structuration qui caractérisent le récit sadien. Le château de Roissy fixe les limites d'un cadre spatial tandis que la clôture temporelle est illustrée par les règles strictes qui régissent la vie quotidienne des prisonnières volontaires : «Le jour durant, vous ferez telle corvée qu'on vous confiera pour la tenue de la maison [...]. Le fouet ne vous sera appliqué qu'entre le coucher et le lever du soleil. Mais outre celui qui vous sera donné par qui le désirera, vous serez punie du fouet le soir pour manquement à la règle dans la journée. » (p. 36-37) Seule la clôture d'action diverge, car le désir amoureux d'O n'est pas toujours suivi de sa satisfaction en raison des absences répétées de l'amant. Le caractère différé du désir 
Alexandra DESTAIS, «L'érographie littéraire féminine : la frénésie sexuelle maîtrisée? », dans J. Delorme et Cl. Labrosse (dir.), «Écriture du corps, corps de l'écriture : la dialectique du corps-écriture dans le roman francophone au XX siècle », @nalyses, hiver 2008

féminin relève davantage de l'attente amoureuse propre à la linea amoris (Brulotte, p. 39) que de l'aisance érographique.

Au plan narratif, comme le souligne Anne-Marie Dardigna (p. 104), la mise à distance du corps d'O est nécessaire pour que se déroule le procès érographique qui consiste en la sujétion sexuelle d'une femme. Convoitée par le désir masculin, $\mathrm{O}$ ne peut endosser la responsabilité de l'acte énonciatif. Au corps contraint, lié par des chaînes, muet, dépossédé, correspond un récit filtré par une voix narrative, c'est-àdire un récit à la troisième personne. Le statut "allodiégétique » (Brulotte, p. 250) adopté parfois par la narration permet de mettre à distance le corps de l'héroïne et par conséquent de solliciter la pulsion voyeuriste du lecteur. L'intimité de l'héroïne importe alors moins que l'action qu'elle subit. Par exemple, dans ce passage, $O$ sert d'alibi à l'entrée du lecteur dans la scène :

Elle se tordait avec une telle frénésie pour échapper aux morsures des lanières qu'elle tournoyait presque sur elle-même, devant le poteau, car la chaînette qui la retenait était longue et donc un peu lâche, bien que solide. [...] On prit le parti, après avoir en effet arrêté un instant, de ne recommencer qu'une fois une corde passée autour de la taille [...]. (1954, p. 34)

Pourtant, l'usage de la troisième personne est un trompe-l'œil. Cette mise à distance narrative ne relève pas seulement d'une «fonction idéologique » (Dardigna, p. 105) qui signifie l'abandon de l'identité de sujet. Elle sert aussi à éviter le soupçon autobiographique à une époque où un auteur féminin est amené à utiliser des écrans protecteurs (dont le pseudonyme) afin de se prémunir contre la censure juridique, la stigmatisation familiale et/ou professionnelle, le scandale intellectuel. Cependant, tandis que certains récits érographiques choisissent l'emboîtement narratif et la multiplication des «je » afin d'éviter la confusion des instances et de miner la position centralisatrice d'un narrateur unique, Pauline Réage opte pour un seul niveau narratif, d'où la simplicité formelle d'Histoire d'O. Le déroulement linéaire de l'histoire, la narration au passé, l'unicité du point de vue servent un récit de quête qui reconduit le schéma narratif traditionnel. Dans ce parcours évolutif, l'érotisme n'est qu'un moyen d'accès à l'objet ultime de la quête: la destruction totale et la mort. L'évolution de l'état psychologique d'O, 
Alexandra DESTAIS, «L'érographie littéraire féminine : la frénésie sexuelle maîtrisée? », dans J. Delorme et Cl. Labrosse (dir.), «Écriture du corps, corps de l'écriture : la dialectique du corps-écriture dans le roman francophone au XX siècle », @nalyses, hiver 2008

qui passe de la honte à une fierté insensée, importe en réalité davantage que l'intensité des ébats sexuels.

Afin de montrer sa solidarité avec $\mathrm{O}$, la voix narrative utilise parfois le corps de celle-ci comme le foyer à partir duquel le lecteur visualise la scène. En effet, dans certaines séquences, la mise à distance d'O est compensée par l'exercice d'un point de vue interne qui crée une soudaine proximité avec le personnage grâce à la coïncidence entre sa vision et la nôtre. Par exemple, dans «Les amants de Roissy», la privation temporaire de la vue par la pose d'un bandeau permet au lecteur d'épouser les sensations de l'héroïne. De même, parce que l'appréhension de l'action en cours ne s'effectue pas par le canal visuel, le groupe des hommes de Roissy est dépersonnalisé. La privation de la vue entraine en effet une appréhension tactile et fragmentaire des hommes qui manipulent le corps d'O : « une main lui prit un sein, une bouche saisit la pointe de l'autre» (p. 31). O n'est donc pas le seul personnage de l'histoire à être objectivé. L'inégalité thématique est compensée par une égalité formelle qui reflète la complicité entre le maître et l'esclave. Mais tandis que la voix narrative confère à $\mathrm{O}$ un statut de personne à part entière en rendant accessibles ses sentiments, ses émotions et ses pensées, les personnages masculins sont réduits à leur praxis.

Le récit de Pauline Réage met donc en scène un personnage ambivalent et complexe, à la fois mi-sujet, mi-objet. $O$ n'est pas réductible au stéréotype de la «femme-objet». Contrairement à la règle d'impersonnalité qui régit le récit sadien, Histoire d'O nous propose une héroïne dotée d'une substance psychologique propre. Si, dans la plupart des récits érographiques, un semblant de fil narratif permet d'éviter le sentiment de monotonie dû au principe de répétition de la séance sexuelle, dans Histoire d'O, les contraintes formelles spécifiques à une narration classique se mêlent aux contraintes formelles spécifiques à l'économie de la séance érographique. Le récit de Réage est en effet formé d'une intrigue où l'héroïne vise à demeurer aimée de son amant, puis à mourir à ellemême, d'où un double objectif qui ne peut être atteint qu'au prix de la traversée d'épreuves touchant directement le corps vivant. Ce dernier forme l'objet de la quête du désir masculin alors qu'il constitue pour 
Alexandra DESTAIS, «L'érographie littéraire féminine : la frénésie sexuelle maîtrisée? », dans J. Delorme et Cl. Labrosse (dir.), «Écriture du corps, corps de l'écriture : la dialectique du corps-écriture dans le roman francophone au XX siècle », @nalyses, hiver 2008

l'héroïne le moyen par lequel elle prouve son amour. À ce double statut prêté au corps correspond une double économie narrative, alternant entre le point de vue externe, où le personnage se manifeste comme argument excitant de la séance érographique, et le point de vue interne, qui permet au sujet de la quête de susciter une identification par sympathie.

Les contraintes spécifiques au texte sadien ont donné naissance à un certain mode d'organisation de l'écriture érographique que nous retrouvons en faible proportion dans le livre de Réage, "conte de fées » pour adultes qui relate les diverses étapes d'une métamorphose. Est-ce à dire que les écrivaines sont trop éprises des sortilèges de la linea amoris pour les échanger contre une représentation mécanique des corps au sein d'une narration distanciée qui fait fi de la personne?

\section{La maîtrise du corps sensuel chez Jean(ne) de Berg}

Les règles de structuration du discours érographique ont été reconduites chez Jean(ne) de Berg, pseudonyme qui désigne en réalité Catherine Robbe-Grillet, épouse du Nouveau Romancier et auteure tout récemment d'un petit carnet perdu où elle raconte quelques-unes de ses séances sadomasochistes. Placé sous le sceau d'un pseudonyme masculin, son premier récit, L'Image (1956), est dédié à "Pauline Réage » et préfacé par les initiales «P.R», ce qui, comme le souligne Pascal Pia, " pourrait faire croire que cette préface est de l'auteur de l'Histoire d'O» (p. 356). En réalité, la préface a été écrite par Alain Robbe-Grillet et se manifeste comme un droit de réponse à celle de Jean Paulhan, Le Bonheur dans l'esclavage (1954). Elle constitue le miroir textuel qui réfléchit la préface à Histoire d'O tout en la contrariant puisque tandis que Paulhan met en avant, non sans provocation, le goût des femmes pour l'esclavage, «P.R » dément cette conception jugée naïve. Reste que mené à la première personne, L'Image opte pour le point de vue interne d'un narrateur masculin à la fois témoin attentif et acteur de la scène sadomasochiste.

Chez cette écrivaine, la mise en place d'un scénario constitue le préalable à des séances sadomasochistes dont l'origine réside moins en 
Alexandra DESTAIS, «L'érographie littéraire féminine : la frénésie sexuelle maîtrisée? », dans J. Delorme et Cl. Labrosse (dir.), «Écriture du corps, corps de l'écriture : la dialectique du corps-écriture dans le roman francophone au XX siècle », @nalyses, hiver 2008

un désir sexuel qu'en un projet artistique né d'une décision intellectuelle. Car, selon Catherine Robbe-Grillet, le plaisir d'une telle construction scénique est essentiellement «cosa mentale», comme elle l'a souligné elle-même lors d'un débat littéraire organisé à l'Université de Caen. Les participants font tantôt partie du public, tantôt partie d'un spectacle soigneusement orchestré et assimilé parfois à un «ballet» (Robbe-Grillet, 2007, p. 144). Prêtresse de cérémonies soigneusement élaborées, Jean(ne) de Berg réintègre les règles du texte sadien en organisant un espace, en fixant un programme, en réglant en cours d'action les postures, en visant l'efficacité maximale de la scène, en «coupant court aux attendrissements» (1956, p. 156). Dans Cérémonies de femmes (1985), la voix narrative annonce en ces termes l'objet du récit :

J'ai choisi de raconter quelques cérémonies emblématiques, prises dans un répertoire plus vaste qui tourne souvent autour de mises en scène ritualisées où les immobilisations, les silences, les jeux sur la vue (masques, miroirs, lumières), la mise à distance suggèrent moins l'orgie que le tableau vivant, même s'ils ne s'y réduisent pas. (1985, p. 94)

Dominée par le principe d'ordre, la scène de Jean(n)e de Berg fait naître également l'impression d'un «minutage» (Barthes, p. 31). Cependant, tandis que l'ordre érographique sadien vise la performance sexuelle, l'auteure de L'Image utilise le pouvoir de suggestion du sexuel sans le réaliser, ce dernier étant "suspendu, différé » (Berg, 1985, p. 32). De même que dans La Vénus à la fourrure (Sacher-Masoch, 1870), la dynamique scénique importe moins que le "tableau vivant», "les postures hiératiques et figées », "ses rites, ses décors de chapelle, le fétichisme de ses objets » (Berg, 1985, p. 11). L'indication brève que «la scène marche», que «le tableau s'arrange» (Barthes, p. 31) ne suffirait pas à une écrivaine qui aime à s'attarder sur les modalités de cette mise en place au point d'accorder à la préparation du scénario autant d'attention narrative qu'à son exécution.

Au sein de cet univers, la fièvre sensuelle est maîtrisée à l'extrême. Si l'esclave volontaire ne peut s'empêcher de ressentir les effets du fouet ou des caresses savantes et de les exprimer par un râle, une plainte, une défaillance, il est néanmoins « une couche de chair» (Berg, 1985, 
Alexandra DESTAIS, «L'érographie littéraire féminine : la frénésie sexuelle maîtrisée? », dans J. Delorme et Cl. Labrosse (dir.), «Écriture du corps, corps de l'écriture : la dialectique du corps-écriture dans le roman francophone au XX siècle », @nalyses, hiver 2008

p. 35) qui ne déborde pas les limites assignées à son rôle et qui se pétrifie volontiers dans l'attente de nouvelles directives :

Lui, il n'a pas bougé. Aveugle ou pas, il ne bougera pas, à moins qu'une voix féminine ne lui en donne l'ordre. Il n'a même pas de ces mouvements infimes, rétablissements instinctifs qui poussent, par exemple, à prendre appui sur une jambe puis sur l'autre pour éviter l'ankylose. Il semble pétrifié. Cette statue a pourtant la peau tiède, le cœur qui bat. (1985, p. 134)

La maîtresse de cérémonie veille à mettre un terme aux élans spontanés de la «victime» de même qu'elle veille au contrôle de ses propres émotions. Si elle n'est pas totalement insensible, sa « frénésie » est cependant "sèche, dominée », pareille à «la chasseresse qui a atteint sa proie » (1985, p. 155). Aurait-on ici un exemple d'érographie féminine aussi apathique que le précédent sadien?

Absent du texte sadien et inscrit explicitement dans Histoire d'O, le thème amoureux apparaît néanmoins en filigrane chez Jean(n)e de Berg. Il se laisse deviner soit dans les brefs interstices où l'extrême contraction du rituel érotique se dénoue et laisse place à l'expression affective pure, soit dans l'investissement du bourreau à l'égard de sa victime et dans l'obéissance muette de la victime à l'égard de son bourreau. Par exemple, dans L'Image, masqué par le rite, le trouble amoureux de Claire pour le narrateur se dévoile parfois, mais il est vite réprimé par un redoublement du supplice à l'égard de la jeune Anne, ce qui permet de détourner sur l'esclave l'énergie érotique adressée en réalité au narrateur. Si le tabou d'O est sa propre jouissance, celui de Claire réside dans cet aveu de fragilité extrême qu'est le trouble d'un désir sensuel authentique. Cependant, l'antinomie entre la sensualité amoureuse et le jeu sadomasochiste qui vise à la maîtriser, voire à l'évacuer au profit du seul plaisir cérébral est levée à la fin du récit. Bien qu'il se réapproprie les principales données de l'érotisme noir et prête au personnage féminin les attributs de la maittrise autrefois réservés au libertin au regard froid, l'auteure renverse le rapport de forces préalablement institué. L'équilibre duel traditionnel est restauré au nom de l'amour. La dominatrice Claire quitte son masque pour se livrer corps et âme au narrateur. Le jeu de rôles laisse place au masochisme naturel de Claire et aux pulsions sadiques de Jean au sein 
Alexandra DESTAIS, «L'érographie littéraire féminine : la frénésie sexuelle maîtrisée? », dans J. DelORME et Cl. LABROSSE (dir.), «Écriture du corps, corps de l'écriture : la dialectique du corps-écriture dans le roman francophone au XX siècle », @nalyses, hiver 2008

d'une scène érotico-amoureuse conventionnelle: «Lorsque je l'ai pénétrée pour de bon, elle s'est aussitôt mise à gémir, m’appelant par mon prénom et me répétant qu'elle m'aimait...» (Berg, 1956, p. 183)

\section{Le sensualisme d'Alina Reyes : quelle maîtrise?}

«Réenchanter la sexualité » par la mise en valeur de l'autre jouissance, tel est le noble but que poursuit Alina Reyes depuis le succès du Boucher (1988). Chez cette écrivaine, l'érotisme est conçu comme un mode d'inscription poétique dans l'existence et comme une voie d'accès au sacré. Ceci n'est pas sans rappeler La Confession anonyme de Suzanne Lilar, publié en 1960, qui cherche à contrer l'érotisme de l'abject (illustré selon l'auteure par Bataille, Sade, mais aussi par Histoire d'O) via un «ars amatoria » (De Decker, 1986) fondé sur une érotique dite «féminine ». Cependant, tandis que Lilar fait du désir sensuel la force motrice permettant d'accéder à une connaissance supérieure où la chair, purifiée, devient même superflue, Alina Reyes situe le sacré au sein même de l'étreinte amoureuse sur laquelle elle semble broder à l'infini. Que l'érotisme des corps soit vécu ou fantasmé, la narratrice se plait à concilier dans un même passage l'évocation explicite des parties sexuelles de l'anatomie humaine et la transposition poétique de l'organique direct :

\footnotetext{
Ami, sens ma bite dure brûlante et droite, touche ma chatte soyeuse noire détrempée, envisage mon cul tabou, plonge en ma bouche affamée assoiffée, repose et spasme en mes mains recueillantes, dévoile, montre-toi aux trous brillants de mes pupilles, mes tympans vibrent comme des harpes éoliennes, les cordes vocales s'étirent et s'étendent du bout de mes vingt doigts au sommet de mon crâne. Je ne puis te chanter les colombes de tes yeux ni les prairies de ta peau mon âme est trop brutale, c'est une âme de mon temps et elle a trop de sexes pour n'être pas violente, c'est une âme du début des temps et elle a trop de sexes pour n'être pas crue. (2004, p. 227)
}

Mené à la première personne, le récit met l'accent sur la vie intérieure d'une femme amoureuse dont l'amant forme davantage le prétexte à l'exaltation d'un désir sans fin qu'un objet d'amour singulier à conquérir. Le corps de l'héroïne est un corps saturé de signes expressifs qui traduisent le plus souvent son appétit vital et son enthousiasme face aux merveilles de la vie et de l'amour. Non 
Alexandra DESTAIS, «L'érographie littéraire féminine : la frénésie sexuelle maîtrisée? », dans J. DelORME et Cl. LABROSSE (dir.), «Écriture du corps, corps de l'écriture : la dialectique du corps-écriture dans le roman francophone au XX siècle », @nalyses, hiver 2008

hermétique, ce corps est un lieu de réceptivité extraordinaire qui accueille et se nourrit des sensations les plus variées. Non limité à un jeu de rôle qui contraindrait ses élans spontanés, le corps fait l'objet d'une représentation qui cherche à le saisir dans sa totalité organique, sensorielle, érogène et non pas dans quelques lieux fétiches. Semblant déborder de toutes parts les limites ordinaires assignées au vivant, le corps féminin ne cesse de convoiter une essence masculine, «l'être que je ne suis pas », «à travers des livres, des images, des hommes, à travers tous ces corps »:

Ce n'est pas moi, ce sont mes mains. Mes lèvres, mes yeux, mes narines. C'est ma peau, et beaucoup plus que ma peau, mes nerfs, mes muscles, mes ongles, mes dents. Qui veulent toucher, happer, éprouver, respirer cette présence-là, ce corps viril organisé de façon cryptique autour de ce qu'on appelait au Moyen Âge le septième membre : le phallus. (2004, p. 92)

Cette omniprésence thématique du corps lyrique dans La Chasse amoureuse est soutenue par une structure formelle lâche, contrairement aux récits érographiques que nous venons d'étudier. Le chant d'amour de ce «roman» mêle les lettres érotiques les plus ardentes à des poèmes, les descriptions d'une enfance habitée par des visions et celles de la nature complice d'une joie intime inépuisable. Seule une division en huit sections le structure. Là encore, c'est la référence corporelle qui fournit le thème de chaque chapitre: «l'odorat», "le goût», "l'ouie », «le toucher», " la vue ». Il s'agit ici moins des étapes de la linea amoris, où le visus forme au contraire la première étape (la reconnaissance réciproque) de la geste amoureuse, que d'une référence intertextuelle à la tapisserie de La Dame à la licorne. Il n'en est pas tout à fait de même de Sept nuits, où la linea amoris est plus fidèlement reconduite (Reyes, 2005). L'auteure nous propose une carte du Tendre érotico-amoureuse. Centrée sur la relation duelle et sur le rapprochement progressif des corps masculin et féminin, elle reproduit les termes du schéma amoureux canonique. Mais étant de nature sexuelle et limités dans le temps, les obstacles instaurent un subtil strip-tease qui relève de l'ordre érotique, où le corps mi-objet, misujet " prend le temps de se mettre en désir et d'être mis en désir » et où c'est à travers le corps de l'autre que se lisent « son âme et sa vie, la façon dont son âme traversait la vie » $(2005$, p. 22). Ce statut corporel 
Alexandra DESTAIS, «L'érographie littéraire féminine : la frénésie sexuelle maîtrisée? », dans J. Delorme et Cl. Labrosse (dir.), «Écriture du corps, corps de l'écriture : la dialectique du corps-écriture dans le roman francophone au XX siècle », @nalyses, hiver 2008

mixte est soutenu par une certaine organisation formelle. À la durée totale de l'histoire correspond en effet un découpage en sept chapitres dont chacun progresse vers la jointure amoureuse. Annoncée par le titre, la clôture temporelle est aussi accordée à une clôture spatiale. La chambre d'hôtel permet d'abriter le couple du tumulte de la vie extérieure et de recréer dans cet espace neutre les noces charnelles d'un homme et d'une femme sans identité autre que celle de leur sexe. L'homme et la femme ne prennent la mesure de leur existence qu'au sein de cet espace clos. Sans identité sociale, ils sont réduits à leur masculinité et à leur féminité, celle-ci étant figurée par les artifices qui apprêtent le «je » féminin avant chaque rendez-vous nocturne. Ils ne sont pourtant pas des outils qui servent de prétexte à la mise en place de la scène érographique. L'effet de réel naît des manifestations d'un corps extrêmement sensible en raison du désir fou qui l'habite. Les termes de l'organique direct abondent pour décrire cette folie amoureuse qui s'empare du corps féminin lorsqu'elle découvre le corps de son amant.

Cependant, le désir s'accroît en proportion des contraintes qui retardent sa satisfaction. L'interdit formulé par l'amant, «Demain, a-til dit. La première nuit, il ne faut pas se toucher...» (2005, p. 14) diffère la jouissance réciproque et fournit donc à la pérennité narrative sa justification thématique. De même que le roman d'amour vit des obstacles qui s'opposent à la réunion heureuse des amants, le récit d'Alina Reyes use de l'attente amoureuse de ses personnages pour susciter un intérêt romanesque. Les obstacles sont ici créés artificiellement et partie intégrante de l'acte charnel lui-même. Ils remplacent les entraves externes d'ordre social et les entraves internes d'ordre psychologique que connaissent les héros et héroïnes des romans d'amour. Ce n'est pas l'étreinte amoureuse qui est soumise à des reports successifs car, dès la seconde nuit, les amants se touchent, mais c'est le « coït» qui forme la finalité du récit, son dénouement et sa consécration. L'acte est ici consubstantiel à la déclaration d'amour : il ne la précède pas comme dans La Confession anonyme ni ne la sanctionne comme dans Anna Karénine. 
Alexandra DESTAIS, «L'érographie littéraire féminine : la frénésie sexuelle maîtrisée? », dans J. Delorme et Cl. LABRosse (dir.), «Écriture du corps, corps de l'écriture : la dialectique du corps-écriture dans le roman francophone au XX siècle », @nalyses, hiver 2008

\section{De la souple architecture amoureuse au dispositif orgiaque de Catherine Millet}

Ainsi, l'étude de ces quelques références confirme qu'il existe une corrélation entre un certain imaginaire du vivant et les contraintes formelles chargées de mettre celui-ci en scène. La représentation du corps féminin, que celle-ci repose sur un principe de réduction sadomasochiste bridant son élan sensuel ou sur un principe d'expansion vitale né du désir amoureux, est soutenue par des procédés cherchant tantôt à le mettre à distance, tantôt à pénétrer son secret. Les contraintes formelles ne visent pas seulement à représenter un personnage féminin "objet» au sein d'une scénographie érotique dont il serait le faire-valoir. Elles servent aussi à mettre en valeur le corps lyrique et à orienter le lecteur vers une activité herméneutique. En effet, les signes affichés par le corps renvoient à une facette de l'intimité psychique du personnage qui reste à déchiffrer en relation avec l'ensemble de l'histoire.

De plus, le primat du couple et l'influence plus ou moins accentuée de la linea amoris chez ces écrivaines empêchent la mise en place d'un système rigoureux. La charpente structurale est moindre que dans les récits où l'interaction entre les personnages, dépassant la dyade, nécessite des agencements complexes, des règles de combinaisons, un ordre interne strict. Au sein du champ de l'érographie féminine contemporaine, seule Catherine Millet semble illustrer le dispositif orgiaque (2001). L’obsession du nombre, la représentation morcelée des corps masculins réduits à leurs parties génitales, le goût de la série, l'attention prêtée aux détails d'une posture définissent ce récit «autobiographique» dont l'autre particularité est d'être délesté du motif amoureux.

\section{Bibliographie}

AlEXANDRIAN, Sarane. 1989, Histoire de la littérature érotique, Paris, Seghers. 
Alexandra DESTAIS, «L'érographie littéraire féminine : la frénésie sexuelle maîtrisée? », dans J. Delorme et Cl. LABROSSE (dir.), «Écriture du corps, corps de l'écriture : la dialectique du corps-écriture dans le roman francophone au XX $\mathrm{X}^{\mathrm{e}}$ siècle », @nalyses, hiver 2008

APOLLINAIRE, Guillaume. 1973, Les Onze Mille verges, Paris, Pauvert.

BARTHES, Roland. 1980a, Sade, Fourier et Loyola, Paris, Seuil, coll. « Points Essais ».

BERG, Jeanne de. 1956, L'Image, Paris, Les Éditions de Minuit ;

—. 1985, Cérémonies de femmes, Paris, Grasset.

Berthelot, Francis. 1997, Le Corps du héros. Pour une sémiologie de l'incarnation romanesque, Paris, Nathan.

Brulotte, Gaëtan. 1998a, Euvres de chair, figures du discours érotique, Paris, L'Harmattan.

Castillo Durante, Daniel. 1996, «Politiques du proverbe chez Sade : du stéréotype à la parole du loup », Carrefour, vol. XVIII, $n^{\circ}$ 2, Ottawa, Éditions Legas, p. 5-62.

DARDIGnA, Anne. 1980b, Les Châteaux d'Éros ou les infortunes du sexe des femmes, Paris, Maspero.

DECKER, Jacques de. 1986, «La Confession anonyme : une ars amatoria », Cahiers Suzanne Lilar, Paris, Gallimard.

DESTAIS, Alexandra (organisation/animation)/LADROUE Marc (mise en scène), Désirs de femmes, «débat littéraire théâtralisé », Maison de l'étudiant, Université de Caen, 26 mai 2005 : avec Alina Reyes, Catherine Robbe-Grillet, Gaëtan Brulotte, Julien CENDRES.

Mandiargues, André Pieyre de. 1955, « Le feu, le fer, la nuit de l'âme », Critique, juin.

MARIE-THÉRÈSE. 1947, «Vie d'une prostituée », Les Temps modernes, décembre, $\mathrm{n}^{\circ} 27$; 
Alexandra DESTAIS, «L'érographie littéraire féminine : la frénésie sexuelle maîtrisée? », dans J. Delorme et Cl. LABROSSE (dir.), «Écriture du corps, corps de l'écriture : la dialectique du corps-écriture dans le roman francophone au XX $\mathrm{X}^{\mathrm{e}}$ siècle », @nalyses, hiver 2008

—.1948, Vie d'une prostituée, Paris, René Bertelé, 1948.

Millet, Catherine. 2001, La Vie sexuelle de Catherine M., Paris, Seuil.

PIA, Pascal. 1998b, «Histoire d'O », dans Les Livres de l'enfer, du $X V I^{e}$ siècle à nos jours, Paris, Fayard, p. 356.

Plante, Christine. 1989, La Petite Sœur de Balzac, Paris, Seuil.

RÉAge, Pauline. Histoire d'O. 1954, Paris, Pauvert.

—. 1969, Retour à Roissy; précédé de Une Fille amoureuse, Paris, Pauvert;

—.1975, Histoire d'O; précédé de Le Bonheur dans l'esclavage par Jean Paulhan, Paris, Pauvert, coll. «Le livre de Poche ».

ReYes, Alina. 1988, Le Boucher, Paris, Seuil.

- 2004, La Chasse amoureuse, Paris, Robert Laffont;

—. 2005, Sept Nuits, Paris, Robert Laffont.

RobBe-GriLlet, Catherine, Le petit carnet perdu, Paris, Fayard, 2007.

SACHER-MASOCH. 1999 [1870], La Vénus à la fourrure, Paris, Mille et une Nuits.

SADE, le marquis de. 1998c [1904], Les Cent Vingt Journées de Sodome ou l'école du libertinage, dans Euvres I, Paris, Gallimard.

TolstoÏ, Léon. 1952, Anna Karénine, Paris, Gallimard. 